\title{
Zentrenbildung in der Allgemein- und Viszeralchirurgie - Notwendigkeit oder Trend?
}

\author{
Formation of Centers in General and Abdominal Surgery - \\ Necessity or Trend?
}

Autoren

Institut

\section{S. M. Freys, C. Strube, A. Friedemann, A. Franke}

DIAKO Ev. Diakonie-Krankenhaus Bremen, Chirurgische Klinik, Kompetenzzentrum Chirurgische Koloproktologie, Darmkrebszentrum Bremen West, Bremen, Deutschland

\section{Schlüsselwörter \\ - Zentrenbildung \\ - Zertifizierung \\ - Qualitätsmanagement \\ - Allgemeinchirurgie \\ - Viszeralchirurgie}

\section{Key words}

- creation of clinical centres

- certification

- quality management

- general surgery

- abdominal surgery
Bibliografie

DOI http://dx.doi.org/

10.1055/s-0031-1283830

Online-Publikation: 7.12.2011

Zentralbl Chir 2013; 138: 29-32

(c) Georg Thieme Verlag KG

Stuttgart · New York

ISSN 0044-409X

\section{Korrespondenzadresse}

Prof. Dr. med.

Stephan M. Freys

DIAKO Ev. Diakonie-

Krankenhaus Bremen,

Chirurgische Klinik

Gröpelinger Heerstr. 406-408

28239 Bremen

Deutschland

Tel.: 0421/61021101

Fax: 0421 / 61021129

s.freys@diako-bremen.de

\section{Zusammenfassung \\ $\nabla$}

Mit Einführung eines einheitlichen fallpauschalenbasierten Vergütungssystems im deutschen Gesundheitssystem hat sich eine Mentalität der Qualitätsorientierung und der Überprüfung medizinischer Behandlungsergebnisse entwickelt. Natürliche Folgen waren die Ausgestaltung medizinischer „Zentren“ auf sehr unterschiedlichem Niveau und konsequenterweise Institutionen, Instanzen und Organisationen zur Überprüfung der formalen und inhaltlichen Qualifikation und Qualität dieser Zentren. Zertifizierungen auf ebenfalls sehr unterschiedlichem Niveau wurden entwickelt, um eine Restrukturierung der teilweise sehr divergenten Zielrichtungen zu erzielen. In diesem Übersichtsartikel erfolgt eine kritische Bewertung der aktuellen Entwicklung von Zentren in der Allgemein- und Viszeralchirurgie in Deutschland.

\section{Einleitung}

\section{$\nabla$}

Das bundesdeutsche Gesundheitssystem hat sich in den vergangenen 10 Jahren durch Einführung der DRG-Systematik grundlegend gewandelt. Nicht medizinische Inhalte sondern ökonomische Belange sind der wesentliche Motor und der Gradmesser des Erfolgs. Die DRG-Systematik ist ein hervorragendes pauschales Abrechnungssystem, welches über Erfolg und Misserfolg einer Abteilung, einer Klinik oder eines gesamten Krankenhauses entscheidet. Parallel zu der Entwicklung dieses noch immer zu verbessernden, vorrangig ökonomischen Notwendigkeiten Rechnung tragenden Gruppierungssystems wurde das Prinzip der Qualitätsmessung und -überprüfung im Gesundheitswesen etabliert. Mit dem Wechsel in der Vergütungssystematik weg von der Aufwandsvergütung („Input-Orientierung“) hin zu einer Fallvergütung bzw. betriebswirtschaftlich gesehen zu einer Produktvergütung („Output-

\section{Abstract \\ $\nabla$}

The introduction of the DRG (diagnosis-related groups) system as basis for reimbursement in the German health-care system has led to a mentality of quality orientation and verification of therapeutic results. An immediate result was the formation of medical "centres" on rather different levels and consequently the inauguration of institutions, authorities, and organisations to review these centres. Finally, a range of certifications was installed in order to stratify the rather diverse aims of different centres. This review critically evaluates the current situation in the field of general and abdominal surgery in Germany.

Orientierung“) wurde zunehmend die Notwendigkeit erkannt, Qualitätsindikatoren zur Beurteilung dieses medizinischen Produktes festzulegen, zu messen und zu überprüfen. Die natürliche Weiterentwicklung des Systems zielt gegenwärtig auf die Möglichkeit ab, komplexe Strukturen zur Vergütung des gesamten Behandlungsergebnisses („Outcome-Orientierung“) einzuführen. Diese Vergütungsformen bestehen derzeit teilweise nebeneinander, wobei die Ergebnisvergütung die Fallvergütung nicht ersetzen, sondern ergänzen soll ( $\bullet$ Abb.1).

\section{Qualitätsorientierung \\ $\nabla$}

Die Gefahr einer vorrangig betriebswirtschaftlichen Dominanz bei den vielschichtigen Entscheidungsprozessen im Gesundheitswesen wurde rechtzeitig erkannt und durch das neue Stilmittel „Qualitätsmessung“ ergänzt. Durch wann 


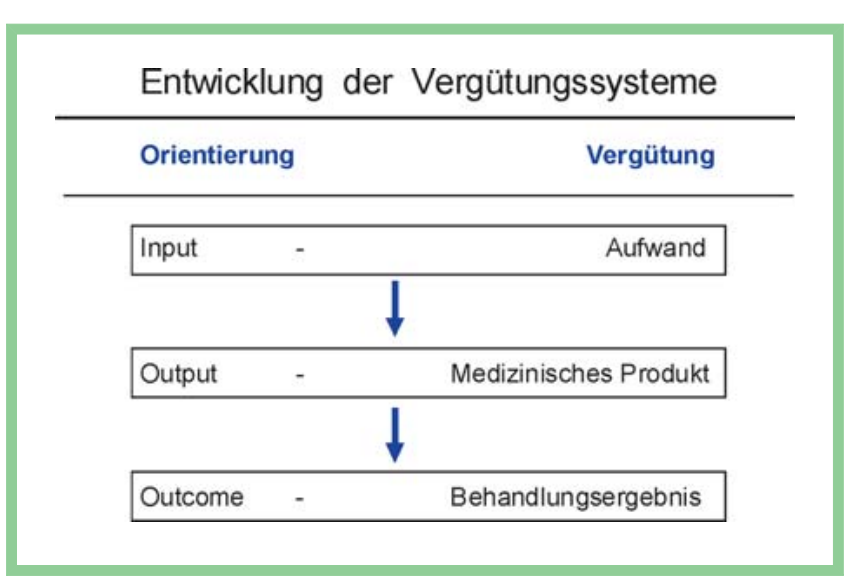

Abb.1 Entwicklung der Vergütungssysteme.

immer möglich evidenzbasierte Daten soll die Qualität einer Behandlung durchleuchtet und objektiviert, bestenfalls sogar optimiert werden. Eine Vielzahl von Qualitätsinitiativen, primär entlehnt aus der Produktionssystematik der Industrie, hat unser Gesundheitssystem überzogen und wurde teilweise durch selbst ernannte Qualitätsspezialisten, teilweise durch akademische Institutionen und neu geschaffene komplexe Verbundsysteme etabliert. Auf diese Weise entwickelten sich an vielen Stellen im Gesundheitswesen Zertifizierungsinitiativen ganz unterschiedlichen Ausmaßes, wobei sowohl einzelne Verfahren, organisatorische Einheiten, Praxen, Kliniken und Krankenhäuser bzw. sektorübergreifende ambulante und stationäre Leistungserbringer einem neu geschaffenen Zertifizierungsprozess unterworfen wurden [1 -3]. Das gemeinsame Bestreben der sehr heterogenen Zertifizierungsinitiativen ist eine höchst mögliche Objektivierung von Qualitätsindikatoren. Sehr unterschiedlich ist bei den verschiedenen Systemen noch die tatsächlich gemessene Qualität $[4,5]$, teilweise wird nur eine Strukturqualität bestimmt, komplexere Systeme erlauben die Messung der Prozessqualität, letztendlich anzustreben ist die weit darüber hinausgehende Messung der Ergebnisqualität, wobei hier eine kurzfristige von einer mittel- bis langfristigen Ergebnisqualität unterschieden wird.

\section{Zentrenbildung \\ $\nabla$}

\section{Systematik}

Natürliche Konsequenz einer zunehmenden ökonomischen Orientierung und der Akzeptanz von Qualitätsprinzipien war die Neueinrichtung oder Ausgestaltung von Zentren. Der Begriff Zentrum ist hierbei schwierig, weil keine klaren inhaltlichen, räumlichen oder juristischen Normen existieren. Zentren können sehr unterschiedliche Ziele verfolgen, sie können qualitäts- oder profitorientiert sein, als Marketinginstrument dienen oder einen sektor- und berufsgruppenübergreifenden Verbund darstellen. Heruntergebrochen auf das umfangreiche Gebiet der Allgemeinund Viszeralchirurgie haben sich in den vergangenen Jahren im Wesentlichen 3 parallel voneinander entwickelte, teilweise ineinander greifende oder sogar aufeinander aufbauende Systematiken einer Zentrenbildung entwickelt. Jede dieser Systematiken zielt darauf ab, medizinische Inhalte zum Gradmesser des Erfolgs und der Qualität einer medizinischen Institution zu erheben - in Konkurrenz zu der heute vorrangig ökonomischen Bemessung eben dieser medizinischen Institution. Die Wechselwirkung die- ser beiden grundverschiedenen Belange bestimmt Inhalt und Ausmaß der 3 möglichen Systematiken einer Zentrenbildung: An erster Stelle wurden Zentren entwickelt, die oft auf persönlicher und regionaler Einzelinitiative die Notwendigkeiten von ökonomischen und medizinischen Belangen versuchten zu vereinbaren. Es entwickelten sich vornehmlich mit medizinischen Inhalten ausgeprägte Zentren, die sich mit einzelnen Krankheitsbildern, Ablauforganisationen oder thematisch orientierten Inhalten befassten („Bauchzentrum“, „klinische Pfade“, „interdisziplinäre Netzwerke“). Diese oft in Eigenregie organisierten Strukturen unterlagen meist keinem Zertifizierungsverfahren. Sie waren vielerorts Wegbereiter für eine zunehmend organisatorisch und wissenschaftlich fundierte Ausgestaltung von zunächst mit strukturellen Fragestellungen befassten Systemen. So entwickelten die Fachgesellschaften in der Deutschen Gesellschaft für Chirurgie mit den Möglichkeiten wissenschaftlich überprüfbarer Daten und teilweise auf dem Boden evidenzbasierter Leitlinien eine 2.Systematik zertifizierter Zentren für die dem jeweiligen Fachgebiet zugehörigen Krankheitsbilder. Es wurden Zertifizierungsorganisationen und -gesellschaften gegründet, die in einem strukturierten Prozess Struktur-, Prozessund Ergebnisqualität in unterschiedlichem Ausmaß messen und überprüfen und es wurden Kataloge zur Eingangsvoraussetzung und Leistungserbringung definiert. Gegenwärtig hat die Deutsche Gesellschaft für Allgemein- und Viszeralchirurgie (www. dgav.de) hier zumindest numerisch eine Führungsrolle in den wissenschaftlichen Fachgesellschaften und im Verbund der Deutschen Gesellschaft für Chirurgie (www.dgch.de) mit aktuell 175 zertifizierten Zentren in 7 Spezialgebieten übernommen ( $\bullet$ Tab. 1). Parallel und übergeordnet hat das Bundesministerium für Gesundheit mit Erstellung des nationalen Krebsplans eine 3. Systematik entwickelt, in dem „Zentren der onkologischen Versorgung" definiert und in 3 Zertifizierungsstufen eingeteilt wurden [6]. Aktuell sind deutschlandweit 681 Zentren als Organkrebszentren, gynäkologische Krebszentren, onkologische Zentren und modulare Zentren in Anbindung an ein bestehendes Organkrebszentrum durch die Firma OnkoZert zertifiziert [7] $(\bullet$ Tab.2).

\section{Qualitätsindikatoren}

Alle Bestrebungen einer wie auch immer gearteten Zentrenbildung im Rahmen der oben genannten 3 Systematiken sollten auf eine Verbesserung der medizinischen Behandlungsqualität abzielen. Voraussetzungen für eine Qualitätsmessung sind zugrundeliegende hochwertige Leitlinien, aus denen sich gesicherte Zertifizierungsschritte bei der Etablierung eines Zentrums ableiten lassen. Wenn der enorme Aufwand einer Messung von Struktur-und Prozessqualität, insbesondere aber von Ergebnisqualität erfolgen soll, so ist dies nur dann sinnvoll, wenn die Resultate dieser Messungen bei festgestellten Abweichungen auf das Management bzw. die Prozesse der zugrunde liegenden Systematik rückwirken können. Das Ziel einer solchen sehr komplexen Organisation ist eine einheitliche und vergleichende Veröffentlichung von Qualitätskennzahlen als Gradmesser für das jeweilige System. Beispiele hierfür sind die German Inpatient Quality Indicators (G-IQI) der HELIOS-Gruppe oder die QSR-Methodik (Qualitätssicherung der stationären Versorgung mit Routinedaten) des wissenschaftlichen Institutes der AOK (WIdO) [8]. Eine solche Intensivierung der Qualitätsorientierung kann letztendlich zu einer qualitätsbasierten Vergütung führen, indem bei- 


\begin{tabular}{|c|c|c|c|c|c|}
\hline Fach & Zentrum & Anzahl & AG & Anzahl & \multirow{8}{*}{$\begin{array}{l}\text { Tab. } 1 \text { Zertifizierte Zentren der } \\
\text { Deutschen Gesellschaft für Chi- } \\
\text { rurgie DGCh (Stand: 23.05.2011). }\end{array}$} \\
\hline DGT & Thoraxzentrum & 9 & CA-CP & 74 & \\
\hline DGU & Traumanetzwerk & 10 & CA-ES & 40 & \\
\hline DGTHG & Herzchirurg. Zentrum & 84 & CA-MIC & 22 & \\
\hline \multirow[t]{2}{*}{ DGG } & \multirow[t]{2}{*}{ Gefäßzentren } & \multirow[t]{2}{*}{105} & CA-LGP & 13 & \\
\hline & & & CA-ADIP & 12 & \\
\hline \multirow[t]{2}{*}{ DGAV } & \multirow[t]{2}{*}{ CA-ADIP, -CP, -EK, -ES, -LGP, -MIC, -OGI } & \multirow{2}{*}{$175 \rightarrow$} & CA-EK & 11 & \\
\hline & & & CA-OGI & 3 & \\
\hline
\end{tabular}

(Legende: DGT - Deutsche Gesellschaft für Thoraxchirurgie, DGU-Deutsche Gesellschaft für Unfallchirurgie, DGTHG - Deutsche Gesellschaft für Thorax- und Herzchirurgie, DGG - Deutsche Gesellschaft für Gefäßchirurgie, DGAV - Deutsche Gesellschaft für Allgemeinund Viszeralchirurgie, DGKCH - Deutsche Gesellschaft für Kinderchirurgie, DGNC - Deutsche Gesellschaft für Neurochirurgie, DGOOCDeutsche Gesellschaft für Orthopädie und Orthopädische Chirurgie, DGPRÄC - Deutsche Gesellschaft für Plastische, Rekonstruktive und Ästhetische Chirurgie, DGMKG - Deutsche Gesellschaft für Mund-, Kiefer- und Gesichtschirurgie, CACP-Chirurgische Arbeitsgemeinschaft für Coloproktologie, CAES - Chirurgische Arbeitsgemeinschaft für Endoskopie und Sonographie, CAMIC - Chirurgische Arbeitsgemeinschaft für Minimal Invasive Chirurgie, CALGP- Chirurgische Arbeitsgemeinschaft für Leber-, Galle-, Pankreaschirurgie, CAADIP- Chirurgische Arbeitsgemeinschaft für Adipositaschirurgie, CAEK - Chirurgische Arbeitsgemeinschaft für Endokrine Chirurgie, CAOGI - Chirurgische Arbeitsgemeinschaft Oberer Gastrointestinaltrakt

Tab. 2 Zertifizierte Zentren OnkoZert.

\begin{tabular}{|lcr}
\hline Zentrum & Anzahl & \multicolumn{1}{c}{ Stand } \\
\hline Brustkrebs & 257 & \\
\hline Darmkrebs & 202 & \\
\hline Prostatakarzinom & 70 \\
\hline Hautkrebs & 33 \\
\hline Lungenkrebs & 24 \\
\hline Gynäkologisches Krebszentrum & 57 \\
\hline Onkologisches Zentrum & 24 \\
\hline Modul Pankreaskarzinom & 14 \\
\hline Modul Kopf-Hals-Tumore & 0 \\
\hline Modul Neuroonkologie & 0 \\
\hline
\end{tabular}

spielsweise eine ergebnisorientierte Modifikation der Basisfallwerte im DRG-System eingeführt würde.

Voraussetzung für eine breite Akzeptanz und vor allem Verständlichkeit der unterschiedlichen Qualitätsinitiativen ist eine Vereinheitlichung der Anforderungen an mögliche Qualitätsindikatoren ( Tab.3). Diese teilweise sehr komplexen Begrifflichkeiten müssen zum einen nicht nur den Leistungserbringern und -überprüfern, sondern vor allem auch dem Laienpublikum verständlich gemacht werden. Nur so können zukünftig Patienten mit den teilweise noch sehr individuellen Ranking-Systemen einzelner Anbieter (Krankenhäuser, Praxen, Krankenkassen) lernen umzugehen. Klinik-Barometer, Krankenhausspiegel und Ranglisten in der Laienpresse sind hier bisher nur unvollständige Messinstrumente. Zudem müssen die Qualitätsindikatoren eine zuverlässige Messbarkeit von außen, d.h. durch unabhängige Institutionen ohne ökonomisches Eigeninteresse gewährleisten. Hand in Hand mit dieser Entwicklung ergeht die Voraussetzung einer Manipulationsresistenz durch den individuellen Leistungserbringer, d.h. das betrachtete und zertifizierte Zentrum selbst.

Tab.3 Anforderungen an Qualitätsindikatoren.
- adäquate medizinische Aussagekraft
- ausreichender wissenschaftlicher Bezug
- ökonomische Relevanz
- angemessener Aufwand bei Erfassung
- Möglichkeit der zeitnahen Erfassung
- Sektorrelevanz
- Manipulationssicherheit
- systemimmanente Anreizwirkungen

\section{Probleme}

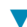

Diese enorme Entwicklung einer Zentrenbildung mit immer noch sehr unterschiedlichen Systematiken ist an keiner Stelle im Gesundheitssystem vergütungsrelevant. Alle Initiativen erfolgen vor dem Hintergrund einer enormen Leistungsbereitschaft der jeweils organisatorisch verantwortlichen Ärzte, Kliniken und assoziierten Praxen niedergelassener Ärzte. Der oft immense zeitliche, personelle, und emotionale Aufwand ist gegenwärtig allein eine immaterielle Investition in die Zukunft, ohne dass es messbare Erfolgsparameter aus ökonomischer Sicht gibt. Selbstverständlich ist Grundlage einer jeglichen selbst auferlegten oder übernommenen Zertifizierungsaktivität das Bestreben einer Qualitätsverbesserung medizinischer Inhalte, wobei allenthalben genauso selbstverständlich eine Optimierung der Marktpositionierung mitschwingt.

Gegenwärtig liegen die Probleme einer flächendeckenden oder institutionsübergreifenden Qualitätsmessung vornehmlich im strukturellen Bereich. Es fehlen derzeit wirksame Controlling-Instrumente, nur für eine begrenzte Anzahl von Diagnosen und Krankheitsbildern kann eine Ergebnisqualität in überschaubaren Zeiträumen gemessen werden und ein wesentliches Handicap ist eine tatsächlich sektorübergreifende, d.h. sowohl im ambulanten wie auch im stationären Bereich durchzuführende Realisierung einer geforderten Qualitätsmessung. Darüber hinaus spielen wiederum ökonomische Probleme eine nicht zu unterschätzende Rolle, da gegenwärtig keine Anreize zu einer flächendeckenden Umsetzung einer Qualitätsmessung existieren, wie dies beispielsweise eine Honorierung durch die Kostenträger darstellen würde. Gerade dieser letzte Aspekt ist extrem kritisch zu bewerten, da hier die offensichtliche Diskrepanz der systeminhärenten Interessenkonflikte zwischen Leistungserbringern und Kostenträgern vordergründig wird.

Es ist nur schwerlich vorstellbar, welche Institution, welche Partei, Leistungserbringer oder Kostenträger vorrangig die Regie in einer adäquaten Leistungsmessung übernehmen soll. Die derzeit existierenden, teilweise extrem komplexen und immer noch sehr heterogenen Zertifizierungsrichtlinien der unterschiedlichen Anbieter (wissenschaftliche Fachgesellschaften, Deutsche Krebsgesellschaft, Krankenhauskonzerne) tragen in ebenso unterschiedlichem Ausmaß zu einer Qualitätsverbesserung bei. Eine inhaltliche Vereinheitlichung und gemeinsame Verabredung zur Stratifizierung der gemessenen Qualitätsindikatoren ist hier vorrangiges Ziel. Sicherlich ist eine Messung und Überprüfung der 
medizinischen Qualität anhand überprüfbarer Qualitätsindikatoren ein grundnotwendiges Ziel der medizinischen Leistungserbringer, um neben dem Primat der ökonomischen Relevanz den Stellenwert der medizinischen Kernkompetenz zum Gradmesser von Entscheidungsprozessen im Gesundheitssystem zu machen.

\section{Fazit}

$\nabla$

Gerade in der Allgemein- und Viszeralchirurgie als Fachgebiet mit einer frühzeitig ausgestalteten „Zertifizierungsmoral“ sollte es gelingen, auf dem Boden der aktuell erreichten Systematik dem Ziel der Betonung einer medizinischen Kernkompetenz Rechnung zu tragen. Hier sollten bei formaler Sichtweise abgestufte Zertifizierungsmodelle zur Aufrechterhaltung der Arbeitsfähigkeit von Kliniken der Grund- und Regelversorgung entwickelt werden. Ein Scheitern an notwendigerweise zu erbringenden Mindestmengen darf nicht zu einer fehlgeleiteten Zentrenbildung mit für Patienten unzumutbaren Wegbelastungen führen. Fiktive Mindestmengen und gut gemeinte, aber zu hoch gestaltete Qualitätshürden dürfen umgekehrt nicht zu einem Qualitätsverlust in der Breite führen.

In mentaler Hinsicht muss die jeweils leistungserbringende Einheit bei der Etablierung eines Zentrums in gleichem Maße eine ehrliche Trennung zwischen Marketing-Initiativen und medizinischer Grundversorgung ermöglichen. Der scheinbare Erfolg einer Zentrenbildung ist immer gegenüber dem Preis der Vernachlässigung des Regelversorgungsauftrages zu bewerten.
Aus inhaltlicher Sicht sollte demnach stets die Kongruenz zur Kernkompetenz der Klinik gewahrt bleiben, wenn eine Zentrenbildung gefordert wird. Die formale Ausgestaltung einer Zentrenbildung sollte sich somit stets auch an die lokalen personellen Gegebenheiten anknüpfen, eine Zentrenbildung kann immer nur das Produkt der Einzelfaktoren darstellen und nicht die Summe aller potenziell wünschenswerten Vorstellungen.

Interessenkonflikt: Nein

\section{Literatur}

1 Hohmann UH. Vom Unsinn der Zertifizierung. Der Chirurg (BDC) 2002; 41: 303

2 Niedermeier H, Eckstein HH, Noppeney T et al. Zertifizierung von Gefäßzentren durch die Deutsche Gesellschaft für Gefäßchirurgie. Chirurg BDC 2007; 46: 215-216

3 Asmuth J, Diel F, Thoma E. Erfahrungen mit der Zertifizierung von Arztpraxen. Chirurg BDC 2007; 46: 229-230

4 Post S. Qualitätsmanagement: Zertifizierung: Fluch oder Segen? Zentralbl Chir 2010; 135: 1-2

5 Musholt TJ. Eodem vadit Endokrine Chirurgie: Zertifizierte Kompetenzzentren weisen in die Zukunft. Zentralbl Chir 2010; 135: 201 - 202

6 Bundesministerium für Gesundheit (BMG). Nationaler Krebsplan Handlungsfeld 2: Weiterentwicklung der onkologischen Versorgungsstrukturen und der Qualitätssicherung (08.06.2011). Im Internet: www.bmg.bund.de Stand 08.10.2011

7 Onkozert. Zertifizierungsinstitut der Deutschen Krebsgesellschaft. Im Internet: www.onkozert.de Stand 23.05.2011

8 Klauber J, Geraedts M, Friedrich J et al. Krankenhaus-Report 2011. Stuttgart: Schattauer; 2011 